\title{
Enfermedad fúngica invasora en pacientes hemato-oncológicos y receptores de trasplante de precursores hematopoyéticos bajo la perspectiva de los criterios diagnósticos EORTC/MSG
}

\author{
Ricardo Rabagliati B., Gino Fuentes L., Ana María Guzmán D., Eric Orellana U., \\ Jorge Oporto C., Igor Aedo C., Marcelo Garrido S. y Bruno Nervi N.
}

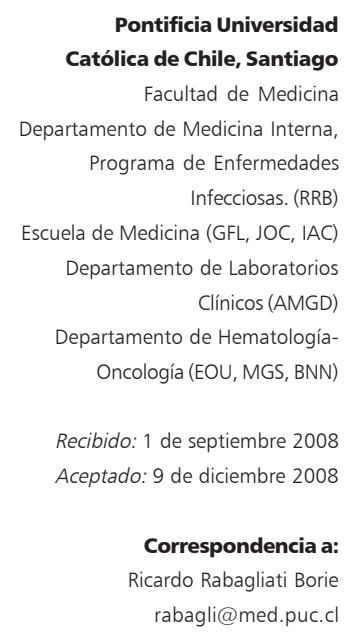

Correspondencia a: rabagli@med.puc.cl

\section{Invasive fungal disease in hemato-oncological and hematopoietic stem cell transplantation patients from Hospital Clinico Universidad Catolica, Santiago-Chile using revised EORTC/MSG diagnostic criteria}

Introduction: Invasive fungal disease (IFD) is a severe complication occurring mostly in haematooncological (H-O) patients and hematopoietic stem cell transplant (HSCT) receptors. Our aim was to describe the IFD occurring in our H-O and HSCT patients according to the EORTC/MSG revised criteria. Patients and Methods: IFD surveillance was performed in adult patients of the Hospital Clínico Universidad Catolica, Santiago, Chile, from January 2004 to January 2008. Results: A total of 41 IFD episodes were identified in 39 patients; mean age was $46.6 \pm 9.9$ years, and $87.8 \%$ and $12.2 \%$ occurred in H-O and HCTS patients respectively. $15 / 41(36.6 \%)$ episodes were proven, $36.6 \%$ probable and $11 / 41(26.8 \%)$ possible. In $26(63.4 \%)$ episodes aspergillosis was diagnosed (20 pulmonary, 3 sinus, 1 laryngeal and 1 case with pulmonary and cerebral involvement). In 7 patients $(17.1 \%$ ) candidiasis was diagnosed, 5 with a proven bloodstream infection and 2 with possible hepatosplenic candidiasis; mucormycosis was diagnosed in 4 (9.8\%) Fusarium infection was demonstrated in 2 patients (4.9\%), and Mucor and Aspergillus pulmonary coinfection and Alternaria sp rhino-sinusitis in one patient each. The frequency of IFD among febrile neutropenic patients was $26.2 \%$ and $6.4 \%$ in $\mathrm{H}-\mathrm{O}$ and HSCT receptors respectively. The overall mortality was $36 \%$. Conclusions: Aspergillosis is the most common IFD infection among H-O patients and HSCT receptors in our center. Candidiasis followed although only in H-O patients most probably because of routine use of antifungal prophylaxis in HSCT recipients. Continuous surveillance is required to develop local guidelines and to evaluate antifungal strategies in different clinical scenarios.

Key words: Febrile neutropenia, hematopoietic stem cell transplantation, mycoses, etiology, cancer, chemotherapy complications.

Palabras clave: Neutropenia febril, trasplante de precursores hematopoyéticos, micosis, etiología, cáncer, complicaciones de quimioterapia.

\section{Introducción}

$\mathrm{D}$ urante los últimos 10 años se han logrado importantes avances en el reconocimiento e identificación de las infecciones fúngicas invasoras en pacientes hemato-oncológicos (H-O) y receptores de trasplante de precursores hematopoyéticos (TPH) gracias a la incorporación de nuevas herramientas diagnósticas tales como marcadores serológicos de antígeno de galactomanano y $1 \rightarrow 3-\beta$-D-glucano ${ }^{1-3}$, avances en el uso e interpretación de los estudios de imágenes ${ }^{4}$ y el desarrollo de criterios diagnósticos de consenso internacional.

Entre estos se encuentran los criterios del "Grupo Colaborativo de Infecciones Fúngicas Invasoras de la
Organización Europea para la Investigación y Tratamiento del Cáncer" en conjunto con su par de E.U.A. el "Grupo de Estudio de Micosis del Instituto Nacional de Alergias y Enfermedades Infecciosas" conocidos por la sigla EORTC/MSG. En su primera versión publicada en $2002^{5}$, clasificó los episodios de infección fúngica en categorías posible, probable o demostra$d a$, definiendo un lenguaje homogéneo para ser usado en el diseño de estudios clínicos y la comunicación de resultados en la literatura internacional. Estos criterios de consenso fueron recientemente revisados ${ }^{6}$ e incorporaron el término enfermedad fúngica invasora (EFI) para resaltar la condición de enfermedad producida por la infección fúngica, por lo que en este trabajo preferiremos este término. 
La EFI es causa frecuente de morbilidad y mortalidad en pacientes $\mathrm{H}-\mathrm{O}$ y en receptores de $\mathrm{TPH}^{7-9}$. El desarrollo de EFI en estos pacientes constituye una complicación grave que lleva a prolongar los días de hospitalización, elevar los costos de la terapia médica, retardar el inicio de nuevos ciclos de quimioterapia y exponer a los pacientes a los riesgos de toxicidad e interacciones por el uso de antifúngicos con el consecuente impacto sobre el pronóstico de enfermedad de base $^{9,10}$.

De acuerdo a los datos publicados en la literatura científica internacional, Candida sp y Aspergillus sp son responsables de 80 a $90 \%$ de las EFI observadas en estos pacientes, y en 10 a $20 \%$ se describen EFI por mucorales, Fusarium sp y Scedosporium sp, los cuales constituyen un grupo de hongos emergentes, ya que su identificación ha aumentado en los últimos años. Con menor frecuencia se describen otras especies que presentan importantes dificultades diagnóstico-terapéuticas tales como Trichosporon sp, Rhodotorula sp, Alternaria sp, Bipolaris sp y Curvularia $\mathrm{sp}^{11-13}$.

La importancia relativa de los diferentes tipos de EFI en pacientes $\mathrm{H}-\mathrm{O}$ puede variar en las distintas zonas geográficas, por lo que es de gran utilidad conocer la epidemiología de los diferentes países y en cada centro asistencial, tal como fuese señalado en el Consenso Chileno de Neutropenia Febril (NF) ${ }^{14}$.

En Chile, existen datos en población pediátrica oncológica en quienes las EFI se presentan en aproximadamente $9 \%$ de los niños con NF por cáncer hematológico o de órganos sólidos; candidiasis es la infección más frecuente en este grupo de pacientes ${ }^{15}$. A nuestro conocimiento, no hay datos publicados de la frecuencia y tipo de EFI que afectan a pacientes adultos chilenos con patología $\mathrm{H}-\mathrm{O}$ y/o receptores de TPH.

El objetivo de este trabajo fue describir las EFI diagnosticadas con ayuda de las nuevas herramientas disponibles en pacientes adultos $\mathrm{H}-\mathrm{O}$ y receptores de TPH del Hospital Clínico Universidad Católica de Chile (HCUC) clasificadas bajo la perspectiva de los criterios EORTC/MSG.

\section{Pacientes y Métodos}

El HCUC es un recinto hospitalario de nivel terciario de atención, ubicado en Santiago, donde anualmente se internan alrededor de 25.000 pacientes. Cuenta con 493 camas, de las cuales 58 corresponden preferentemente a cuidados de pacientes oncológicos adultos. Junto al HCUC existe un centro ambulatorio de diagnóstico y terapia del cáncer "Nuestra Señora de la
Esperanza", al que anualmente consultan alrededor de 9.000 pacientes, de los cuales $30 \%$ corresponden a portadores de patología $\mathrm{H}-\mathrm{O}$ (leucemias, linfomas y mielomas) que son derivados al HCUC para procedimientos diagnósticos o terapéuticos incluyendo manejo de infecciones. Por otra parte, en el HCUC se realizan alrededor de $20 \mathrm{TPH}$ al año en pacientes adultos: alogénico, autólogo y de cordón, siendo la indicación más frecuentes la enfermedad H-O. De acuerdo a las guías locales, los pacientes receptores de TPH alogénicos y de cordón reciben fluconazol profiláctico por al menos cuatro semanas post-trasplante, pero no se considera el uso de profilaxis antifúngica de rutina en pacientes sometidos a TPH autólogo o en quimioterapia.

En este escenario se diseñó un estudio de vigilancia de EFI en que se identificaron episodios diagnosticados en pacientes adultos (15 años o más) con enfermedad $\mathrm{H}-\mathrm{O}$, con o sin neutropenia, y receptores de TPH en el período comprendido entre enero de 2004 y enero de 2008. El diagnóstico de EFI fue realizado por el equipo tratante frente a cada caso particular en base a la sospecha clínica.

En los casos identificados se completó una ficha que incluyó antecedentes demográficos y enfermedad de base (patología H-O de base, quimioterapia, tipo de $\mathrm{TPH}$, recuento absoluto de neutrófilos, uso de inmunosupresores $\mathrm{y} / \mathrm{o}$ corticoesteroides); resultados de hemocultivos, cultivo de hongos, identificación de especie (API 32C de Biomerioux ${ }^{\circledR}$ ), búsqueda de antígeno galactomanano circulante (Bio-Rad Platelia ${ }^{\circledR}$ Aspergillus EIA de BioMerieux ${ }^{\circledR}$ ) y antifungigrama (Etest ${ }^{\circledR} \mathrm{Ab}$ de Biodisk $\left.{ }^{\circledR}\right)$; estudio de imágenes e histología cuando estaban disponibles; terapia antifúngica recibida y mortalidad. Los episodios fueron calificados como EFI posible, probable y demostrada de acuerdo a los criterios del consenso EORTC/MSG recientemente revisados $^{6}$ (Tabla 1).

Los resultados de las variables nominales se expresan en porcentajes y de las numéricas en promedio \pm desviación estándar (DS).

\section{Resultados}

Durante el período de estudio se identificaron 41 episodios de EFI en 39 pacientes, 36 (87,8\%) episodios se presentaron en pacientes con patología $\mathrm{H}-\mathrm{O}$, predominantemente pacientes con leucemia, algunos casos en pacientes con linfoma y ningún episodio identificado en pacientes con mieloma. Cinco $(12,2 \%)$ correspondieron a receptores de TPH alogénico y no hubo casos identificados en receptores de TPH de cordón o autólogos. De los 36 episodios en pacientes 
Tabla 1. Resumen de criterios diagnósticos revisados de enfermedad fúngica invasora según consenso EORTC/MSG ${ }^{6}$

\begin{tabular}{|c|c|}
\hline Factores del hospedero & $\begin{array}{l}\text { - Neutropenia } \\
\text { - Receptor de TPH } \\
\text { - Uso de corticosteroides por más de tres semanas } \\
\text { - Uso de inmunosupresores } \\
\text { - Inmunodeficiencia primaria }\end{array}$ \\
\hline Criterios clínicos & $\begin{array}{l}\text { - Enfermedad respiratoria baja (al menos uno): Nódulos con o } \\
\text { sin halo, creciente aéreo o cavidad } \\
\text { - Traqueobronquitis } \\
\text { - Rinosinusitis } \\
\text { Sinusitis en estudio de imágenes con (al menos uno): Dolor agudo, } \\
\text { escara nasal, compromiso de paredes óseas cavidad paranasal } \\
\text { - Infección del sistema nervioso central (al menos uno): Lesiones } \\
\text { focales o reforzamiento meníngeo } \\
\text { - Candidiasis diseminada (al menos uno post-candidemia): Le- } \\
\text { sión en "ojo de buey" en hígado/bazo o exudados en la retina }\end{array}$ \\
\hline Criterios micológicos & $\begin{array}{l}\text { - Directos: tinciones o cultivos positivos } \\
\text { - Indirectos: galactomanano o } 1 \rightarrow 3-\beta d \text {-glucano }\end{array}$ \\
\hline Infección probada & $\begin{array}{l}\text { Tinción y/o cultivo que demuestre levaduras u hongo filamentoso } \\
\text { en sangre o muestra clínica de cavidad estéril o estudio histológico } \\
\text { con evidencia de invasión fúngica }\end{array}$ \\
\hline Infección probable & Factores de hospedero más criterios clínicos y criterios micológicos \\
\hline Infección posible & Factores de hospedero más criterios clínicos sin criterios micológicos \\
\hline
\end{tabular}

Tabla 2. Características generales de 41 episodios de EFI diagnosticados en 39 pacientes durante el período 2004 a 2008. Hospital Clínico Universidad Católica de Chile

\begin{tabular}{|c|c|}
\hline & $\begin{array}{l}n \quad(\%) \\
n=41\end{array}$ \\
\hline Edad, años (promedio \pm DS) & $46,6 \pm 9,9$ \\
\hline Sexo masculino & $24(58,5)$ \\
\hline Episodios en pacientes hemato-oncológicos $(\mathrm{H}-\mathrm{O})$ & $36(87,8)$ \\
\hline Leucemia mieloide aguda & $19(52,8)$ \\
\hline Leucemia linfática aguda & $7(19,4)$ \\
\hline Leucemia linfática crónica & $3(8,3)$ \\
\hline \multicolumn{2}{|l|}{ Otras leucemias } \\
\hline \multicolumn{2}{|l|}{ (células velludas, bifenotípica, } \\
\hline mielomonocítica, mieloide crónica) & $5(13,9)$ \\
\hline Linfomas & $2(5,6)$ \\
\hline Episodios en pacientes receptores de TPH alogénico & $5(12,2)$ \\
\hline \multicolumn{2}{|l|}{ Categoría diagnóstica EORTC/MSG } \\
\hline EFI demostrada & $15(36,6)$ \\
\hline EFl probable & $15(36,6)$ \\
\hline EFI posible & $11(26,8)$ \\
\hline \multicolumn{2}{|l|}{ Etiología EFI } \\
\hline Aspergilosis & $26(63,4)$ \\
\hline Candidiasis & $7(17,1)$ \\
\hline Mucormicosis & $4 \quad(9,8)$ \\
\hline Fusariosis & $2(4,9)$ \\
\hline Aspergilosis más mucormicosis & $1 \quad(2,4)$ \\
\hline Alternariosis & $1 \quad(2,4)$ \\
\hline
\end{tabular}

H-O, $32(88,9 \%)$ se presentaron en pacientes neutropénicos post-quimioterapia. La EFI más frecuentemente identificada fue aspergilosis, seguida por candidiasis. Menos frecuentemente se identificaron episodios de mucormicosis, fusariosis y hubo un caso de EFI por Alternaria sp y otro de co-infección Aspergillus y un mucoral. De acuerdo a los criterios diagnósticos EORTC/MSG ${ }^{6}$, predominaron los casos identificados como EFI demostradas y probables (Tabla 2). En dos casos hubo una segunda EFI identificada, en un caso una aspergilosis probable habiéndose diagnosticado tres meses antes una candidiasis posible y en otro caso una paciente en que se diagnosticó una mucormicosis luego de una aspergilosis probable, totalizando 41 episodios en 39 pacientes.

A modo de estimar la frecuencia de EFI en pacientes $\mathrm{H}-\mathrm{O}$ con NF y en receptores de TPH se consideraron respectivamente los denominadores "Número de episodios de NF en pacientes con cáncer hematológico" a partir de los datos de la vigilancia epidemiológica de NF de nuestro centro y "Número total de TPH" realizados en el HCPUC durante el período enero 2004-enero 2008. Se calculó entonces una frecuencia de $26,2 \%$ en pacientes NF con cáncer hematológico (32 casos de EFI en 122 episodios de NF) y de 6,4\%, en TPH, correspondiente a cinco episodios en $78 \mathrm{TPH}$ durante el período enero 2004-enero 2008 (38 TPH alogénicos, 34 autólogos y 6 cordones).

Específicamente, en el período de estudio se identificaron 26 casos de aspergilosis. Diez y seis de 26 $(61,5 \%)$ correspondieron a pacientes con leucemia mieloide aguda y 17/26 $(65,4 \%)$ a casos probables. En aquellos pacientes con cultivos positivos, se identificó $A$. fumigatus en cuatro casos, A. flavus en tres casos y $A$. terreus en un caso. La localización más frecuente fue la pulmonar. El diagnóstico de aspergilosis demostrada se basó en evidencias histológicas o cultivos de tejido o de muestra de sitio estéril que demostraron presencia de Aspergillus sp. Los casos probables se diagnosticaron en base a las características del hospedero, estudio de galactomanano positivo (índice $\geq 0,5 \mathrm{ng} / \mathrm{ml}$ ) y TAC de tórax que evidenciaba nódulos con signo del halo. Los casos posibles sólo presentaron serología de galactomanano positiva o nódulos pulmonares, con o sin halo (Tabla 3 ).

De los siete episodios de candidiasis, cinco correspondieron a casos clasificados como EFI demostrada a través de hemocultivos que identificaron $C$. albicans (n: 3), C. krusei (n: 1) y C. kefyr (n: 1). En este último caso además se identificaron múltiples lesiones cutáneas que correspondieron a compromiso cutáneo por C. kefyr demostrado por cultivo y estudio histológico. En los otros dos casos el diagnóstico se basó en un cuadro clínico e imágenes tomográficas características 
de candidiasis hepato-esplénica, pero sin confirmación microbiológica, a pesar del estudio con biopsia hepática, por lo que se catalogaron como EFI posibles. Todos los casos de candidiasis corresponden a pacientes H-O en quimioterapia: leucemia linfática aguda (n: 3), leucemia mieloide aguda (n: 2) y linfoma no Hodgkin (dos casos, uno de ellos en paciente con infección por VIH), mientras que entre los receptores de TPH no se identificaron casos de candidiasis.

Los cuatro casos de mucormicosis correspondieron a EFI demostrada, de localización rinosinusal (n: 2), oral (n: 1) y pulmonar (n: 1). En tres casos se identificó Rhizopus orizae. Tres casos correspondieron a pacientes con leucemia mieloide aguda durante $\mathrm{NF}$, uno de ellos estando bajo tratamiento prolongado con voriconazol por una aspergilosis diagnosticada previamente, y un caso correspondió a un paciente receptor de TPH alogénico durante un episodio grave de enfermedad de injerto contra huésped.

Los dos casos de fusariosis correspondieron a EFI demostradas con hemocultivos positivos a Fusarium solani. Se trataba de pacientes con leucemia aguda, en un caso mieloide y en otro leucemia linfática, que cursaban NF. En ambos casos se presentaron lesiones cutáneas múltiples altamente sugerentes de fusariosis; en uno de ellos se realizó estudio histológico y cultivo de una lesión de piel confirmándose el compromiso cutáneo por $F$. solani.

El caso de EFI por Alternaria sp correspondió a una EFI probada de foco rinosinusal en un paciente con leucemia mielomonocítica cursando NF post quimioterapia.

El paciente que presentó co-infección por Aspergillus sp y un mucoral estaba en tratamiento por leucemia de células velludas; se diagnosticó a través del estudio histológico de una biopsia pulmonar obtenida por punción bajo TAC de los nódulos pulmonares.

De los 26 casos de aspergilosis 10 fueron tratados con caspofungina $(38,5 \%)$, cuatro con voriconazol $(15,4 \%)$, siete con asociación de ambos $(26,9 \%)$ y cinco recibieron simultánea o secuencialmente anfotericina-B deoxicolato y caspofungina $(19,2 \%)$. De los siete casos con candidiasis, la terapia inicial fue fluconazol en cinco pacientes; sólo en dos episodios se prefirió iniciar con caspofungina o anfotericina-B deoxicolato. Los casos de mucormicosis fueron tratados con anfotericina B liposomal y desbridamiento quirúrgico; uno de los casos recibió posaconazol en forma prolongada sin evidencias de progresión de la EFI en los ciclos de quimioterapia subsiguientes o durante el TPH alogénico al que fue sometido. Ambos casos de fusariosis recibieron voriconazol. La co-infección por mucoral y $A$. terreus fue tratada con anfotericina B-liposomal más caspofungina. La infección
Tabla 3. Características generales del grupo de 26 casos de aspergilosis. Hospital Clínico Universidad Católica de Chile

\begin{tabular}{|c|c|}
\hline & n $(\%)$ \\
\hline Edad (promedio \pm DS) & $49,1 \pm 16,1$ \\
\hline Sexo masculino & $14(53,8)$ \\
\hline Pacientes hemato-oncológicos & $22(85)$ \\
\hline Leucemia mieloide aguda & $14(63,7)$ \\
\hline Leucemia linfática aguda & $4(21,0)$ \\
\hline Leucemia linfática crónica & $2(9,1)$ \\
\hline Otras leucemias & $2(9,1)$ \\
\hline Linfomas & 0 \\
\hline Pacientes receptores de TPH & $4(15)$ \\
\hline \multicolumn{2}{|l|}{ Categoría diagnóstica EORTC/MSG } \\
\hline Demostrada & $3(11,5 \%)$ \\
\hline Probable & $17(65 \%)$ \\
\hline Posible & $6(23 \%)$ \\
\hline \multicolumn{2}{|l|}{ Diagnóstico } \\
\hline Cultivo positivo & $8(30,8)$ \\
\hline Biopsia con hifas septadas invasoras & $3(11,5)$ \\
\hline Galactomanano positivo $(\geq 0,5)^{*}$ & $15(71)$ \\
\hline TAC pulmonar con nódulos** & $20(76,9)$ \\
\hline TAC pulmonar con nódulos con signo del halo & $9(34,6)$ \\
\hline \multicolumn{2}{|l|}{ Localización*** } \\
\hline Pulmonar & $20(76,9)$ \\
\hline Rinosinusal & $3(11,5)$ \\
\hline Laríngea & $1(3,8)$ \\
\hline Cerebral + pulmonar & $1(3,8)$ \\
\hline
\end{tabular}

Tabla 4. Comparación de EFI entre pacientes

H-O y receptores de TPH. . Hospital Clínico Universidad Católica de Chile

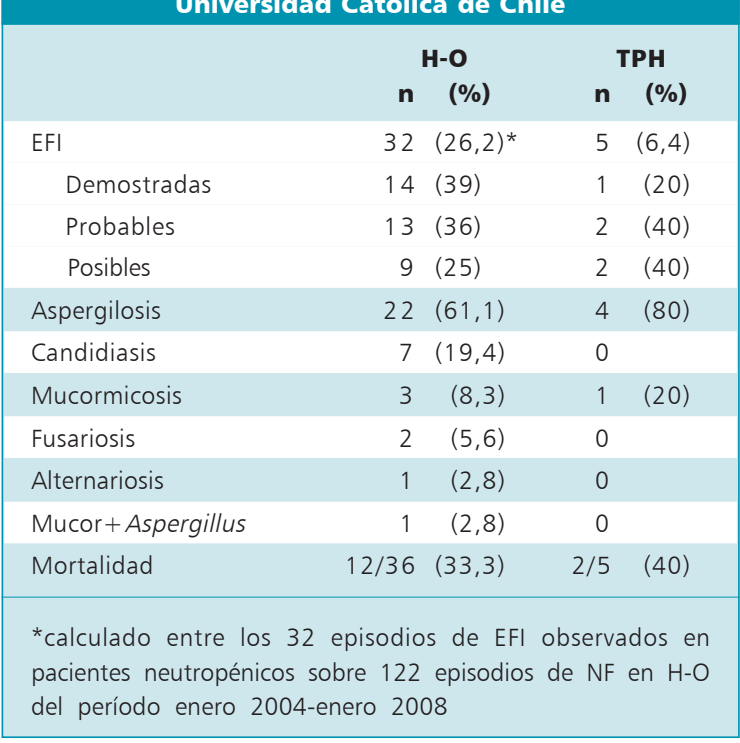


rinosinusal por Alternaria sp se manejó con desbridamiento quirúrgico y voriconazol.

La mortalidad global observada fue de $36 \%$, siendo máxima $(75 \%)$ en los casos de mucormicosis, seguido por aspergilosis (33\%) y luego candidiasis (14\%). En los dos casos de fusariosis se logró su estabilización con el tratamiento antifúngico pero posteriormente fallecieron por leucemia refractaria por lo que su mortalidad no es atribuible a la EFI. No hubo mortalidad asociada al caso de co-infección pulmonar de mucor más Aspergillus ni al caso de alternariosis.

\section{Discusión}

Aspergilosis constituye la EFI más frecuente en nuestro centro en pacientes adultos $\mathrm{H}-\mathrm{O}$, incluyendo pacientes receptores de TPH. Candidiasis, si bien es la segunda en frecuencia, se encuentra relativamente alejada, y entre los hongos emergentes se incluyen los mucorales, Fusarium sp y Alternaria sp.

Este comportamiento de aspergilosis en nuestro centro, corresponde a lo descrito en la literatura médica internacional durante los últimos años, donde se señala que Aspergillus sp, es el hongo más frecuentemente identificado como agente responsable de EFI en adultos y niños con patología $\mathrm{H}-\mathrm{O}^{16-18}$, incluyendo TPH donde se describe con mayor frecuencia en trasplante alogénico (hasta 26\%) y menos frecuentemente en autólogo (2 a $6 \%)^{19}$. Uno de los factores a considerar es que las herramientas diagnósticas actuales como el antígeno de galactomanano y la interpretación de imágenes tomográficas de pulmón facilitan el diagnóstico de aspergilosis, lo cual puede llevar a que ésta se encuentre proporcionalmente más diagnosticada que aquellas EFI que carecen de marcadores serológicos e imagenológicos. Sin embargo, en estudio de autopsias en grandes series en E.U.A. se ha demostrado disminución de la frecuencia de candidiasis y aumento de aspergilosis ${ }^{20}$. Si bien es cierto, podría cuestionarse incluir los casos posibles; precisamente los criterios internacionales consideran que para estudios de tipo epidemiológicos pueden ser incluidos, mientras que para aquellos de efectividad de antifúngicos solamente deben considerarse los probables y demostrados ${ }^{5}$. De todos modos, si en nuestros resultados consideráramos solamente los casos de aspergilosis probable y demostrada, continuaría siendo la primera causa de EFI en nuestros pacientes.

Candidiasis podría estar sub-diagnosticada en esta serie, ya que efectivamente, el estudio microbiológico es de pobre rendimiento ${ }^{21}$. Se describen de cierta utilidad: la presencia de imágenes características de embolias fúngicas en la retina, observadas a través de fondo de ojo, aunque su identificación es infrecuen$\mathrm{te}^{22}$, y las imágenes en "ojo de buey" en la TAC de hígado que orientan a una candidiasis hepato-esplénica $^{23}$. Sin embargo, a diferencia de aspergilosis, no existen marcadores específicos para el diagnóstico de candidiasis. Puede resultar atractivo el uso del marcador serológico $1 \rightarrow 3-\beta$-D-glucano que, si bien no permite discriminar el tipo específico de micosis, su cotejo con otras serologías puede orientar el diagnóstico de $\mathrm{EFI}^{3,24}$. Lamentablemente, el uso de biología molecular no ha logrado avances significativos en el diagnóstico de candidiasis a pesar de múltiples esfuerzos, tanto en pacientes neutropénicos como no neutropénicos ${ }^{25,26}$. Como contrapartida, según datos publicados en la literatura médica internacional, la frecuencia de candidiasis en este tipo de pacientes ha disminuido ${ }^{17}$, lo que podría atribuirse al uso de fluconazol profiláctico. Esto podría explicar la ausencia de candidiasis en los pacientes receptores de TPH incluidos en este estudio, ya que todos ellos recibieron fluconazol por lo menos durante el primer mes post TPH. Otro de los aspectos señalados en la literatura científica corresponde a la emergencia de especies de Candida no-albicans en estos pacientes como las especies predominantes ${ }^{27}$, lo cual tiene implicancias terapéuticas ya que pueden presentar menor susceptibilidad a los azoles. Si bien en nuestros resultados el número de Candida sp identificado fue pequeño, $40 \%$ de las especies identificadas corresponde al grupo no-albicans.

La frecuencia de EFI en pacientes neutropénicos H$\mathrm{O}$ de nuestra serie resultó mayor a la descrita en niños chilenos con $\mathrm{NF}^{15}$, lo que concuerda con el dato conocido de la mayor frecuencia de infecciones fúngicas en adultos que en niños neutropénicos. Entre los pacientes H-O, la EFI se observó principalmente en aquellos con leucemias, hubo baja frecuencia en linfomas y no se diagnosticaron casos en pacientes con mieloma, dato probablemente explicable por su menor frecuencia de neutropenia. Entre los pacientes con TPH que desarrollaron EFI todos habían sido sometidos a trasplantes alogénicos, en cuyo caso las neutropenias son más prolongadas que en el trasplante autólogo. El pequeño número de pacientes sometidos a trasplantes de cordón en este período puede explicar la ausencia de EFI en este grupo aunque no podemos descartar como explicación las limitaciones diagnósticas. Al comparar la frecuencia de EFI determinadas para $\mathrm{H}-\mathrm{O}$ con la de TPH, se observa una menor frecuencia en el grupo TPH; sin embargo, se debe ser cauto al interpretar esta comparación, en vista que en el caso de los episodios de EFI en NF está acotado a los pacientes sintomáticos febriles, a diferencia del grupo TPH que considera a todos los individuos en riesgo aunque hayan estado asintomáticos. 
La utilidad de estudios de la epidemiología de EFI en cada centro permite conocer la situación local, base para planificar estrategias de prevención, guiar el enfrentamiento diagnóstico y la terapia empírica. En forma complementaria se deben considerar los factores de riesgo de $\mathrm{EFI}^{28-32}$ de cuyo análisis se puede decidir la estrategia más apropiada de enfrentamiento del problema frente al paciente. Básicamente las estrategias a definir en cada centro, de acuerdo a este tipo de estudios, son el uso de antifúngicos profilácticos o aquellas que privilegien el uso de antifúngicos en forma más dirigida como ocurre en las estrategias "empírica" y la "terapia anticipada" (del inglés pre-emptive therapy).

El uso de fluconazol demostró disminuir significativamente las infecciones por Candida en pacientes receptores de TPH en estudios clínicos a inicios de los años ' $90^{33}$. A través de meta-análisis comparando antifúngicos profilácticos versus placebo o nada, se ha evidenciado reducción de EFI y de mortalidad relacionada a infección fúngica en pacientes neutropénicos post quimioterapia, pero no de mortalidad general ${ }^{34}$. Tal como se señalaba, el uso de fluconazol en los pacientes con TPH en nuestro centro podría explicar la ausencia de candidemia en estos pacientes. Se ha intentado uso de otros antifúngicos con fines profilácticos: itraconazo ${ }^{35,36}$, anfotericina B liposomal ${ }^{37}$, voriconazo $^{38}$, caspofungina ${ }^{39}$ y micafungina ${ }^{40}$, con beneficios variables sobre sus comparadores, pero el más reciente y atractivo de los estudios fue el uso de posaconazol profiláctico en pacientes $\mathrm{H}-\mathrm{O}$ sometidos a quimioterapia y en pacientes sometidos a TPH con enfermedad de injerto contra huésped, demostrando una disminución estadísticamente significativa de EFI, tanto por levaduras como hongos filamentosos ${ }^{41,42}$. Sin embargo, se desconoce su efecto a mediano y largo plazo en esta población respecto a la emergencia de otros hongos o la aparición de resistencia a fármacos antifúngicos. Resulta preocupante la descripción de emergencia de infecciones por mucorales en pacientes que reciben voriconazol ${ }^{43}$, situación que se presentó en uno de los pacientes de nuestra serie, quien desarrolló infección por un mucoral estando en tratamiento prolongado con voriconazol. Este hecho plantea la duda si será un efecto sobre la ecología de los hongos o la historia natural de pacientes profundamente inmunocomprometidos que sobreviven más en relación a mejores tratamientos.

En cuanto al uso de antifúngicos empíricos, como por ejemplo anfotericina-B deoxicolato en neutropenia persistentemente febril, fue demostrado efectivo en disminuir la frecuencia de EFI según estudios de los años $80^{44}$, pero su uso implica exponer innecesariamente a muchos pacientes a los efectos tóxicos de este fármaco; la alternativa de utilizar anfotericina $\mathrm{B}$ liposomal, con reducido perfil de toxicidad, no es aplicable en la práctica clínica de nuestro medio como tratamiento empírico por sus elevadísimos costos. Más recientemente, en estudios conducidos por Walsh y cols, acerca del uso de antifúngicos empíricos en NF, se demostraron resultados comparables entre caspofungina y anfotericina $\mathrm{B}$ liposomal ${ }^{45}$ no así con voriconazo ${ }^{46}$, esquemas que también presentan limitante por costos. Ante esto, una mejor selección de pacientes para la indicación de antifúngicos empíricos, según factores de riesgo, resulta atractiva para disminuir el número de pacientes expuestos a antifúngicos ${ }^{47}$. Otra alternativa es iniciar terapia antifúngica cuando existan evidencias iniciales de EFI (terapia anticipada); esta estrategia apunta al manejo de aspergilosis que cuenta con herramientas de diagnóstico precoz, dejando fuera otras EFI. No se conoce la efectividad real de esta estrategia ni tampoco si la disminución de uso de antifúngicos empíricos por la elección de una terapia anticipada podría incrementar la frecuencia de otras EFI.

En resumen, en nuestro medio parece razonable limitar la profilaxis antifúngica con fluconazol a los receptores de TPH. Mientras que en los pacientes con NF constituye un área en evolución; la estrategia preferible debe ser evaluada en cada centro tomando en consideración sus datos epidemiológicos, acceso a laboratorio diagnóstico y antifúngicos.

Se debe destacar la baja mortalidad observada en los pacientes de esta serie; podrían estar influyendo diversos factores: se incluyen casos posibles, diagnóstico más precoz en aspergilosis basado en serología e imágenes fácilmente disponibles en nuestro centro y terapias basadas en nuevos antifúngicos reconocidamente más efectivos.

Nuestra serie clínica presenta limitaciones ya que no se realizó un estudio protocolizado de diagnóstico a cada paciente en riesgo, sino que se basó en los resultados obtenidos de exámenes solicitados según la sospecha diagnóstica por el equipo tratante; podría considerarse que existe sub-diagnóstico de casos. Por otro lado, ya se ha mencionado que este estudio podría sobredimensionar la magnitud de frecuencia de aspergilosis al emplear criterios microbiológicos serológicos no disponible para otras EFI pero, considerando que se emplean criterios de consenso aceptados internacionalmente, creemos que los resultados son válidos y en el caso que excluyéramos los casos posibles de aspergilosis ésta sigue siendo la EFI más frecuente en estos pacientes. Tratándose de la experiencia de un solo centro no se pueden extrapolar estos hallazgos a la situación de otros establecimientos del país, pero la concordancia de nuestros resultados con 
lo publicado en la literatura médica internacional sugiere que podría ser una realidad en otros centros en Chile que atiendan pacientes de similares características.

En resumen, con el uso de las técnicas y criterios diagnósticos actualmente disponibles se caracterizó el comportamiento de la EFI en los pacientes H-O y receptores de TPH de nuestra institución, evidenciándose la predominancia de aspergilosis como la infección fúngica más frecuente en ambos grupos de pacientes, seguida de candidiasis, mucormicosis y otras infecciones emergentes en menor incidencia. Es importante contar con estudios nacionales multicéntricos de vigilancia de EFI e identificación de factores de riesgo locales, de cuya evaluación se puedan recomendar, de mejor forma, estrategias farmacológicas y no farmacológicas orientadas a disminuir la frecuencia de EFI en pacientes H-O y receptores de TPH.

\section{Resumen}

Introducción: La enfermedad fúngica invasora (EFI) es una complicación grave en pacientes hematooncológicos (H-O) y receptores de trasplante de precursores hematopoyéticos (TPH). Objetivo: Describir las EFI diagnosticadas en pacientes adultos $\mathrm{H}-\mathrm{O}$ y receptores de TPH de nuestro centro, bajo los criterios diagnósticos revisados de EORTC/MSG. Pacientes y Métodos: Estudio de vigilancia de EFI en pacientes adultos del Hospital Clínico de la Pontificia Universidad Católica de Chile entre enero 2004 y enero 2008. Resultados: Se identificaron 41 episodios de EFI, correspondientes a 39 pacientes: $46,6 \pm 9,9$ años, $87,8 \%$ H-O y $12,2 \%$ TPH. Se documentaron $15 / 41(36,6 \%)$ EFI demostrada, $36,6 \%$ probable y $11 / 41(26,8 \%)$ posible. En 26/41 (63,4\%) se diagnosticó aspergilosis (20 pulmonar, 3 rinosinusal, 1 laríngeo y un caso cerebralpulmonar). En 7/41 (17,1\%) se diagnosticó candidiasis, 5 candidemias y 2 candidiasis hepato-esplénica posibles; 4/41 (9,8\%) correspondió a mucormicosis demostrada ( 2 rinosinusal, 1 oral y 1 pulmonar); en $2 / 41$ (4,9\%) fusariosis; $1 / 41(2,4 \%)$ coinfección pulmonar por mucoral y Aspergillus sp y 1 caso de rinosinusitis por Alternaria sp. La frecuencia de EFI entre pacientes HO con neutropenia febril fue $26,2 \%$ y $6,4 \%$ en los receptores de TPH. La mortalidad global fue de 36\%. Conclusiones: Aspergilosis es la EFI más frecuente en $\mathrm{H}-\mathrm{O}$ y receptores de TPH de nuestro centro. Candidiasis es la segunda EFI en frecuencia; sin embargo, no se documentó entre los pacientes receptores de ТPH, lo que puede relacionarse al uso de antifúngicos profilácticos en este grupo. Es necesaria la vigilancia continua para desarrollar guías clínicas locales y evaluar estrategias de uso de antifúngicos en distintos escenarios clínicos.

\section{Referencias}

1.- Maertens J, Verhaegen J, Lagrou K, Van Eldere J, Boogaerts M. Screening for circulating galactomannan as a noninvasive diagnostic tool for invasive aspergillosis in prolongued neutropenic patients and stem cell trasnplantation recipients: a prospective validation. Blood 2001; 97: 1604-10.

2.- Herbrecht R, Letscher-Bru V, Oprea C, Lioure B, Waller J, Campos F, et al. Aspergillus galactomannan detection in the diagnosis of invasive aspergillosis in cancer patients. J Clin Oncol 2002; 20: 1898-906.

3.- Pazos C, Ponton J, Del Palacio A. Contribution of $(1 \rightarrow 3)$-beta-D-glucan chromogenic assay to diagnosis and therapeutic monitoring of invasive aspergillosis in neutropenic adult patients: a comparison with serial screening for circulating galactomannan. J Clin Microbiol 2005; 43: 299-305.

4.- Caillot D, Couaillier J F, Bernard A, Casasnovas O, Denning D W, Mannone L, et al. Increasing volume and changing characteristics of invasive pulmonary aspergillosis on sequential thoracic computed tomography scans in patients with neutropenia. J Clin Oncol 2001; 19: 253-9.

5.- Ascioglu S, Rex J H, de Pauw B, Bennett J E, Bille J, Crokaert F, et al. Defining opportunistic invasive fungal infections in immunocompromised patients with cancer and hematopoietic stem cell transplants: An International Consensus. Clin Infect Dis 2002; 34: 7-14.

6.- De Pauw B, Walsh,T J, Donnelly P, Stevens D A, Edwards J E, Calandra T, et al. Revised definitions of invasive fungal disease from the European Organization for Research and Treatment of Cancer/Invasive Fungal Infections Cooperative Group and the National Institute of Allergy and Infectious Diseases Mycoses Study Group (EORTC/MSG) Consensus Group. Clin Infect Dis 2008; 46: 1813-21.

7.- Walsh T J, Hiemens J W, Anaissie E. Recent progress and current problems in treatment of invasive fungal infection in neutropenic patients. Infect Dis Clin North Am 1996; 10: $365-400$.

8.- Lin S J, Schranz J, Teutsch S H. Aspergillosis case-fatality rate: systematic review of the literature. Clin Infect Dis 2001; 32: 358-66.

9.- Nucci M, Marr K. Emerging fungal diseases. Clin Infect Dis 2005; 41: 521-6.

10.- Zaoutis T E, Heydon K, Chu J H, Walsh T J, Steinbach W J. Epidemiology, outcomes, and costs of invasive aspergillosis in immunocompromised children in the United States, 2000. Pediatrics 2006; 117: e711-6.

11.- Pagano L, Caira M, Candoni A, Offidani M, Fianchi L, Martino B, et al. The epidemiology of fungal infections in patients with hematologic malignancies: the SEIFEM-2004 study. Haematologica 2006; 91: 1068-75.

12.- Nucci M. Emerging moulds: Fusarium, Scedosporium and Zygomycetes in transplant recipients. Cur Opin Infect Dis 2003; 16: 607-12.

13.- Marr K, Carter R, Crippa F, Wald A, Corey L. Epidemiology and outcome of mould infections in hematopoietic stem cell transplant recipients. Clin Infect Dis 2002; 34: 909-17.

14.- Santolaya M E, Rabagliati R, Bidart T, Paya E, Guzmán A M, Morales R, et al. Consenso Manejo racional del paciente con 
cáncer, neutropenia y fiebre. Rev Chil Infect 2005, 22: S79-113.

15.- Lucero Y, Brücher R, Álvarez A M, Becker A, Cofré J, Enríquez N, et al. Invasive fungal infections in children with cancer, neutropenia and fever, in Chile. Rev Méd Chile 2002; 130: 1139-46.

16.- Marr K, Bowden R A. Fungal infection in patient undergoing blood and marrow transplantation. Transpl Infect Dis 1999; 1: 237-46.

17.- Pagano L, Caira M, Nosari A, Van Lint M T, Candoni A, Offidani M, et al. Fungal infections in recipients of hematopoietic stem cell transplants: results of the SEIFEM B-2004 study-Sorveglianza Epidemiologica Infezioni Fungine Nelle Emopatie Maligne. Clin Infect Dis 2007; 45: 1161-70.

18.- Sung L, Lange B J, Gerbing R B, Alonzo T A, Feusner J. Microbiologically documented infections and infection-related mortality in children with acute myeloid leukemia. Blood 2007; 110: 3532-9.

19.- Singh N, Paterson D L. Aspergillus infections in transplant recipients. Clin Microbiol Rev 2005; 18: 44-69.

20.- Chamilos G, Luna M, Lewis R E, Bodey G P. Chemaly $\mathrm{R}$, Tarrand $\mathrm{J} \mathrm{J}$, et al. Invasive fungal infections in patients with hematologic malignancies in a tertiary care cancer center: an autopsy study over a 15 year period (1989-2003). Haematologica 2006; 91: 986-9.

21.- Anttila V J, Ruutu P, Bondestam S, Jansson S E, Nordling S, Färkkilä M, et al. Hepatosplenic yeast infection in patients with acute leukemia: a diagnostic problema. Clin Infect Dis 1994; 18: 979-81.

22.- Donahue S P, Greven C M, Zuravleff J J, Eller A W, Nguyen M H, Peacock J E Jr, et al. Intraocular candidiasis in patients with candidemia. Clinical implications derived from a prospective multicenter study. Ophtalmology 1994; 101: 1302-9

23.- Karthaus K, Huebner G, Elser C, Geissler R G, Heil G, Ganser A. Early detection of chronic disseminated candida infection in leukemia patients with febrile neutropenia: value of computer-assisted serial ultrasound documentation. Ann Hematol 1998; 77: 41-5.

24.- Obayashi T, Negishi K, Suzuki T, Funata N. Reappraisal of the serum $1 \rightarrow 3 \beta d$-glucan assay for the diagnosis of invasive fungal infections-a study based on autopsy cases from 6 years. Clin Infect Dis 2008; 46: 1864-70.

25.- Ellepola A N, Morrison C J. Laboratory diagnosis of invasive candidiasis. J Microbiol 2005; 43: 65-84.

26.- Moreira-Oliveira M S, Mikami Y, Miyaji M,
Imai T, Schreiber A Z, Moretti M L. Diagnosis of candidemia by polymerase chain reaction and blood culture: prospective study in a high-risk population and identification of variables associated with development of candidemia. Eur J Clin Microbiol Infect Dis 2005; 24: 721-6.

27.- Marr K, Seidel K, White T C, Bowden R A. Candidemia in allogeneic blood and marrow transplant recipients: evolution of risk factors after the adoption of prophylactic fluconazole. J Infect Dis 2000; 181: 309-16.

28.- Latge J P. Aspergillus fumigatus and aspergillosis. Clin Microbiol Rev 1999; 12: 310-50.

29.- Marr K, Carter R A, Boeckh M, Martin P, Corey L. Invasive aspergillosis in allogeneic stem cell transplant recipients: changes in epidemiology and risk factors. Blood 2002; 100: 4358-66.

30.- Richardson M D. Changing patterns and trends in systemic fungal infections. 2005; 56 Suppl 1: i5-i11.

31.- Anaissie E J, Rex J H, Uzun O, Vartivarian S. Predictors of adverse outcome in cancer patients with candidemia. Am J Med 1998; 104: 238-45.

32.- Chayakulkeeree M, Ghannoum M A, Perfect J R. Zygomycosis: the re-emerging fungal infection. Eur J Clin Microb Inf Dis 2006; 25: 215-29.

33. - Goodman J L, Winston D J, Greenfield R A, Chandrasekar P H, Fox B, Kaizer H, et al. A controlled trial of fluconazole to prevent fungal infections in patients undergoing bone marrow transplantation. N Engl J Med 1992; 326: 845-51.

34.- Bow E J, Laverdière M, Lussier N, Rotstein C, Cheang M S, Ioannou S Antifungal prophylaxis for severely neutropenic chemotherapy recipients: a meta analysis of randomized-controlled clinical trials. Cancer 2002; 94: 3230-46.

35.- Nucci M, Biasoli I, Akiti T, Silveira F, Solza C, Barreiros G, et al. A double-blind, randomized, placebo-controlled trial of itraconazole capsules as antifungal prophylaxis for neutropenic patients. Clin Infect Dis 2000; 30: 300-5.

36. - Marr K, Crippa F, Leisenring W, Hoyle M, Boeckh M, Balajee S A, et al. Itraconazole versus fluconazole for prevention of fungal infections in patients receiving allogeneic stem cell transplants. Blood 2004; 103: 1527-33.

37.- Uhlenbrock S, Zimmermann M, Fegeler W, Jürgens H. Ritter J. Liposomal amphotericin $B$ for prophylaxis of invasive fungal infections in high-risk paediatric patients with chemotherapy-related neutropenia: interim analysis of a prospective study. Mycoses 2001; 44: 455-63.
38.- Siwek G T, Pfaller M A, Polgreen P M, Cobb S, Hoth P, Magalhaes-Silverman M, et al. Incidence of invasive aspergillosis among allogeneic hematopoietic stem cell transplant patients receiving voriconazole prophylaxis. Diagn Microb and Infect Dis 2006; 55: 209-12.

39. - Mattiuzzi G N, Alvarado G N, Giles F J, Ostrozky-Zeichner L, Cortés J, O’Brien S, et al. Open-label, randomized comparison of itraconazole versus caspofungin for prophylaxis in patients with hematologic malignancies. Antimicrob Agents Chemother 2006; 50 (1): 143-7.

40.- van Burik J A, Ratanatharathorn V, Stepan D E, Miller C B, Lipton J H, Vesole D H, et al. Micafungin versus fluconazole for prophylaxis against invasive fungal infections during neutropenia in patients undergoing hematopoietic stem cell transplantation. Clin Infec Dis 2004; 39: 1407-16.

41.- Cornely O A, Maertens J, Winston D J, Perfect J, Ullman A J, Walsh T J, et al. Posaconazole vs fluconazole or itraconazole prophylaxis in patients with neutropenia. N Engl J Med 2007; 356: 348-59.

42.- Ullman A J, Lipton J H, Vesole D H, Chandrasekar P, Langston A, Tarantolo S R, et al. Posaconazole or fluconazole for prophylaxis in severe graft-versus-host disease. N Engl J Med 2007; 356: 335-47.

43.- Siwek G T, Dodgson K J, de MagalhaesSilverman M, Bartelt L A, Kilborn S B, Hoth P L, et al. Invasive zygomycosis in hematopoietic stem cell transplant recipients receiving voriconazole prophylaxis. Clin Infect Dis 2004; 39: 584-7.

44.- Pizzo P A, Robichaud K J, Gill F A, Witebsky F G. Empiric antibiotic and antifungal therapy for cancer patients with prolonged fever and granulocytopenia. Am J Med 1982; 72: 101-11.

45.- Walsh T J, Teppler H, Donowitz G R, Maertens J A, Baden L R, Dmoszynska A, et al. Caspofungin versus liposomal amphotericin B for empirical antifungal therapy in patients with persistent fever and neutropenia. N Engl J Med 2004; 351: 1391-402.

46. - Walsh T J, Pappas P, Winston D J, Lazarus H M, Petersen F, Raffalli J, et al. Voriconazole compared with liposomal amphotericin B for empirical antifungal therapy in patients with neutropenia and persistent fever. N Engl J Med 2002; 346: 225-34.

47.- Klastersky J. Antifungal therapy in patients with fever and neutropenia-more rational and less empirical? N Engl J Med 2004; 351: 1445-7. 